\title{
A DIFFERENTIAL GRADED LIE ALGEBRA CONTROLLING THE POISSON DEFORMATIONS OF AN AFFINE POISSON VARIETY
}

\author{
MATEJ FILIP
}

\begin{abstract}
We construct a differential graded Lie algebra $\mathfrak{g}$ controlling the Poisson deformations of an affine Poisson variety. We analyse $\mathfrak{g}$ in the case of affine Gorenstein toric Poisson varieties. Moreover, explicit description of the second and third Hochschild cohomology groups is given for three-dimensional affine Gorenstein toric varieties.
\end{abstract}

\section{INTRODUCTION}

In the last decades differential graded Lie algebras have become a very important tool in deformation theory. A deformation problem is controlled by a differential graded Lie algebra $\mathfrak{h}$ if its corresponding functor of Artin rings is isomorphic to the deformation functor of $\mathfrak{h}$. In characteristic 0 every deformation functor is controlled by a differential graded Lie algebra, due to Quillen, Deligne, Drinfeld and Kontsevich. It is well known that associative non-commutative (resp. commutative) deformations of affine varieties are controlled by the Hochschild (resp. Harrison) differential graded Lie algebra.

In recent years there has been a lot of interest in Poisson deformations, i.e. in deformations of a pair consisting of a variety and a Poisson structure on it (see [5], [7], [9], [10], [1]).

In this paper we construct a differential graded Lie algebra $\mathfrak{g}$ controlling the Poisson deformations of an affine Poisson variety $\operatorname{Spec}(A)$. We see that the Poisson cohomology groups $H^{k}(\mathfrak{g})$ are related to some parts of the Hodge decomposition of Hochschild cohomology groups $\mathrm{HH}^{n}(A)$ (see e.g. [6] for definition of the Hodge decomposition). In the case of affine toric varieties we gave a convex geometric description of these parts in 3 .

The paper is organized as follows. In Section 2 we recall basic deformation theory via differential graded Lie algebras and basic results about the Hodge decomposition of Hochschild cohomology groups. The first main result of this paper is a construction of a differential graded Lie algebra $\mathfrak{g}$ controlling the Poisson deformations, which is done in Section 3. We notice that for the computation of the Poisson cohomology groups $H^{k}(\mathfrak{g})$ parts of the Hodge decomposition of the Hochschild cohomology are relevant (see (10). The Poisson cohomology groups for affine Poisson Gorenstein toric surfaces are computed in Subsection 4.2. Using results in [3] we explicitly compute some parts of the Hochschild cohomology groups in the case of

2010 Mathematics Subject Classification. 13D03, 13D10, 14B05, 14B07, 14M25.

Key words and phrases. Poisson cohomology, Hochschild cohomology, toric varieties, deformations of toric singularities. 
three-dimensional affine Gorenstein toric varieties, which is our second main result (see Theorem 4.11) obtained in Subsection 4.3. This result also reproves and generalizes [1, Theorem 4.4]. In particular, a complete description of the second (which describes the first order deformations) and third Hochschild cohomology group (which contains the obstructions for extending deformations to larger base spaces) is given (see Corollary 4.12).

\section{Preliminaries}

2.1. Deformation theory via differential graded Lie algebras. Let $k$ be a field of characteristic 0 and let $\mathcal{A}$ be the category of local Artinian $k$-algebras with residue field $k$ (with local homomorphisms as morphisms). By $\mathcal{S}$ we denote the category of sets. If not otherwise specified a tensor product $\otimes$ means $\otimes_{k}$. Let $\mathfrak{g}$ be a differential graded Lie algebra (dgla for short). By $\mathfrak{g}^{i}$ we denote the degree $i$ elements of $\mathfrak{g}$.

Definition 1. For a dgla $\mathfrak{g}$ we define the functor $\mathrm{MC}_{\mathfrak{g}}: \mathcal{A} \rightarrow \mathcal{S}$ by

$$
B \mapsto\left\{x \in \mathfrak{g}^{1} \otimes m_{B} \mid d(x)+\frac{1}{2}[x, x]=0\right\} .
$$

$\mathrm{MC}_{\mathfrak{g}}$ is said to be the Maurer-Cartan functor associated to $\mathfrak{g}$. Elements in $\mathrm{MC}_{\mathfrak{g}}(B)$ are the Maurer-Cartan elements of the dgla $\mathfrak{g} \otimes B$.

Definition 2. Let $\mathcal{G}$ denote the category of groups. Let $\mathfrak{g}$ be a dgla and define the functor $\mathrm{G}_{\mathfrak{g}}: \mathcal{A} \rightarrow \mathcal{G}$ given by

$$
B \mapsto \exp \left(\mathfrak{g}^{0} \otimes m_{B}\right),
$$

where exp is the standard exponential functor on Lie algebras. $G_{\mathfrak{g}}$ is said to be the gauge functor associated to $\mathfrak{g}$.

Fix a dgla $\mathfrak{g}$ over $k$; the gauge functor $G_{\mathfrak{g}}$ acts naturally on the Maurer-Cartan functor $\mathrm{MC}_{\mathfrak{g}}$ by the formula

$$
\begin{gathered}
\mathrm{G}_{\mathfrak{g}}(B) \times \mathrm{MC}_{\mathfrak{g}}(B) \rightarrow \mathrm{MC}_{\mathfrak{g}}(B) \\
\left(e^{b}, x\right) \mapsto x+\sum_{n=0}^{\infty} \frac{[b, \cdot]^{n}}{(n+1) !}([b, x]-d(b)) .
\end{gathered}
$$

This action is called the gauge action.

Definition 3. Let $\mathfrak{g}$ be a dgla over $k$. The deformation functor associated to $\mathfrak{g}$ is the functor $\operatorname{Def}_{\mathfrak{g}}: \mathcal{A} \rightarrow \mathcal{S}$ given by

$$
B \mapsto \frac{\mathrm{MC}_{\mathfrak{g}}(B)}{G_{\mathfrak{g}}(B)} .
$$

We say that a dgla $\mathfrak{g}$ controls a functor $F$ if $\operatorname{Def}_{\mathfrak{g}} \cong F$ holds.

2.2. The Hodge decomposition of the Hochschild cohomology. Let $A$ be a finitely generated $k$-algebra. Let $C^{\bullet}(A)$ be the Hochschild cochain complex, i.e., $C^{n}(A)$ is the space of $k$-linear maps $f: A^{\otimes n} \rightarrow A$ (or $A$-module homomorphisms $A \otimes A^{\otimes n} \rightarrow A$ ) with the differential given by

$$
\begin{aligned}
(d f)\left(a_{1} \otimes \cdots \otimes a_{n}\right):= & a_{1} f\left(a_{2} \otimes \cdots \otimes a_{n}\right)+ \\
& \sum_{i=1}^{n-1}(-1)^{i} f\left(a_{1} \otimes \cdots \otimes a_{i} a_{i+1} \otimes \cdots \otimes a_{n}\right)+ \\
& (-1)^{n} f\left(a_{1} \otimes \cdots \otimes a_{n-1}\right) a_{n} .
\end{aligned}
$$


The $n$-th cohomology group of this complex is called the $n$-th Hochschild cohomology group, denoted by $\mathrm{HH}^{n}(A)$. The Lie bracket on $C^{\bullet}(A)[1]$ is coming from the Gerstenhaber bracket $[f, g]$ of $f \in C^{m}(A), g \in C^{n}(A)$, which is defined by

$$
[f, g]:=f \circ g-(-1)^{(m+1)(n+1)} g \circ f \in C^{m+n-1}(A),
$$

where

$$
(f \circ g)\left(a_{1} \otimes \cdots \otimes a_{m+n-1}\right):=
$$

$\sum_{i=1}^{m}(-1)^{(i-1)(n+1)} f\left(a_{1} \otimes \cdots \otimes a_{i-1} \otimes g\left(a_{i} \otimes \cdots \otimes a_{i+n-1}\right) \otimes a_{i+n} \otimes \cdots \otimes a_{m+n-1}\right)$.

The Gerstenhaber bracket equips $C^{\bullet}(A)[1]$ with the structure of a dgla.

Gerstenhaber and Schack described the Hodge decomposition of the Hochschild (co-)homology that we will briefly recall (see [6] for more details). In the group ring of the permutation group $S_{n}$ one defines $s_{i, n-i}$ to be $\sum(\operatorname{sgn} \pi) \pi$, where the sum is taken over those permutations $\pi \in S_{n}$ such that $\pi(1)<\pi(2)<\cdots<\pi(i)$ and $\pi(i+1)<\pi(i+2)<\cdots<\pi(n)$. Let $s_{n}=\sum_{i=1}^{n-1} s_{i, n-i}$. It holds that $C^{n}(A)=C_{(1)}^{n}(A) \oplus \cdots \oplus C_{(n)}^{n}(A)$, where $C_{(i)}^{n}(A)=\left\{f \in C^{n}(A) \mid f \circ s_{n}=\left(2^{i}-2\right) f\right\}$. The Hodge decomposition is

$$
\operatorname{HH}^{n}(A) \cong H_{(1)}^{n}(A) \oplus \cdots \oplus H_{(n)}^{n}(A)
$$

where $H_{(i)}^{n}(A)$ is the $n$-th cohomology of $C_{(i)}(A)$.

We denote the projectors of $\mathrm{HH}^{n}(A)$ to $H_{(i)}^{n}(A)$ by $e_{n}(i)$.

Lemma 2.1. For an element $p \in H_{(2)}^{2}(A)$ and an element $q \in H_{(1)}^{2}(A)$ we have the following:

- the equation $e_{3}(3)[p, p]=0$ is the Jacobi identity, $e_{3}(2)[p, p]=0$

- $[p, q]=e_{3}(2)[p, q]$ and $[q, q]=e_{3}(1)[q, q]$.

Proof. An easy computation, see also [12].

\section{Poisson DEFormations}

Poisson deformations are deformations of a pair consisting of a variety and a Poisson structure on it. Lately there has been a lot of interest in these deformations, see for example results of Namikawa [9, [10, [11] or Kaledin and Ginzburg [7].

Definition 4. A skew-symmetric Hochschild 2-cocycle $p$ (i.e. $p \in C_{(2)}^{2}(A)$ with $d p=0)$ that satisfies the Jacobi identity

$$
p(a \otimes p(b \otimes c))+p(b \otimes p(c \otimes a))+p(c \otimes p(a \otimes b))=0
$$

is called an (algebraic) Poisson structure (or a Poisson bracket). A commutative algebra together with a Poisson bracket is called a Poisson algebra. Its spectrum is called an affine Poisson variety.

Note that $p \in H_{(2)}^{2}(A) \cong \operatorname{hom}_{A}\left(\Omega_{A \mid k}^{2}, A\right)$ (see e.g. [8]), where $\Omega_{A \mid k}^{2}$ is the 2-th exterior power of the module of Kähler differentials. Using Lemma 2.1 we can equivalently define the Poisson structure as an element $p \in C_{(2)}^{2}(A)$ with $d p=$ $e_{3}(3)[p, p]=0$. 
Definition 5. A Poisson deformation of a Poisson algebra $A$ over an Artin ring $B$ is a pair $\left(A^{\prime}, \pi\right)$, where $A^{\prime}$ is a Poisson $B$-algebra and $\pi: A^{\prime} \otimes_{B} k \rightarrow A$ is an isomorphism of Poisson $k$-algebras. Two such deformations $\left(A^{\prime}, \pi_{1}\right)$ and $\left(A^{\prime \prime}, \pi_{2}\right)$ are equivalent if there exists an isomorphism of Poisson $B$-algebras $\phi: A^{\prime} \rightarrow A^{\prime \prime}$ such that it is compatible with $\pi_{1}$ and $\pi_{2}$, i.e. such that $\pi_{1}=\pi_{2} \circ\left(\phi \otimes_{B} k\right)$.

3.1. Dgla that controls the deformation problem. A functor that encodes this deformation problem is

$$
\begin{gathered}
\operatorname{PDef}_{A}: \mathcal{A} \rightarrow \mathcal{S} \\
B \mapsto\{\text { Poisson deformations of } A \text { over } B\} / \sim .
\end{gathered}
$$

In the following we define a dgla that controls the above deformation problem. Consider the Double complex 1.

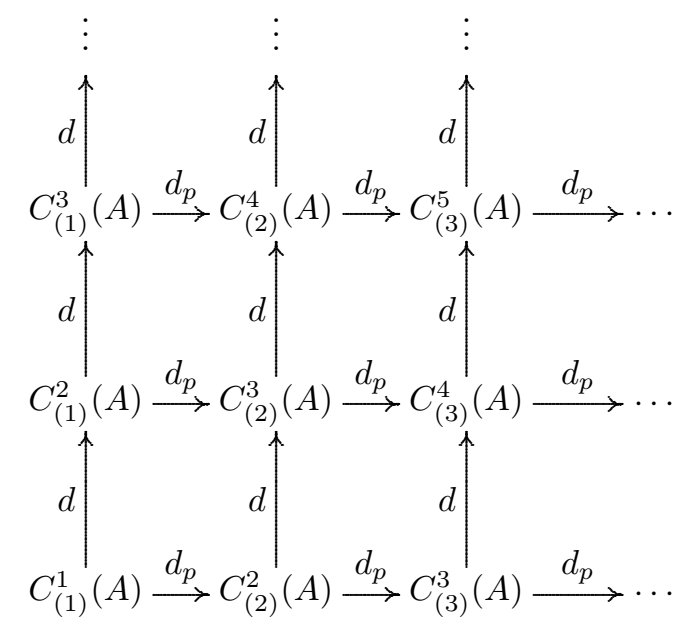

Figure 1. Double complex 1

The map $d_{p}$ is defined as $d_{p}:=-\left[\mu_{p}, \cdot\right]: C^{n}(A) \rightarrow C^{n+1}(A)$, where $\mu_{p} \in C_{(2)}^{2}(A)$ is a Poisson structure on $A$. In the double complex 1 we restrict $d_{p}$ on the chosen domains and codomains. Note that we have $d\left[\mu_{p}, f\right]=\left[\mu_{p}, d f\right]\left(\right.$ since $\left.d \mu_{p}=0\right)$ and thus we really obtain a double complex. We denote its total complex by $D^{\bullet}$.

We define the bracket $[,]_{p}$ on $D^{\bullet}$ as follows: let $C^{n}(A)=C_{(1)}^{n}(A) \oplus \cdots \oplus C_{(n)}^{n}(A)$ and define

$$
\begin{gathered}
{[\cdot, \cdot]_{p}: C^{m}(A) \times C^{n}(A) \rightarrow C^{m+n-1}(A)} \\
{\left[\left(f_{1}, \ldots, f_{m}\right),\left(g_{1}, \ldots, g_{n}\right)\right]_{p}:=\left(\left[f_{1}, g_{1}\right], \ldots, \sum_{i+j=k}\left[f_{i}, g_{j}\right], \ldots,\left[f_{m}, g_{n}\right]\right),}
\end{gathered}
$$

where we restrict $\left[f_{i}, g_{j}\right]$ to $C_{(i+j-1)}^{m+n-1}(A)$.

This bracket defines a dgla structure on $D^{\bullet}[1]$ : the shifted differential $d_{p}[1]$ is equal to $\left[\mu_{p}, \cdot\right]_{p}$ and the shifted differential $d[1]$ is equal to $[\mu, \cdot]_{p}$, where $\mu$ is the commutative multiplication on $A$. We denote the shifted differential of $D^{\bullet}[1]$ by $\tilde{d}$. It is given by $\tilde{d}=\left[\mu+\mu_{p}, \cdot\right]_{p}$. We can immediately check that the bracket $[,]_{p}$ and differential $\tilde{d}$ equip $D^{\bullet}[1]$ with the structure of a dgla. We denote this dgla by $C_{p}^{\bullet}(A)[1]$. 
Remark 1. Note that the Gerstenhaber bracket is in general not graded with respect to the Hodge decomposition and thus the above product is not the Gerstenhaber bracket. From Lemma 2.1 we have $[\mu, \mu]_{p}=[\mu, \mu],\left[\mu, \mu_{p}\right]_{p}=\left[\mu, \mu_{p}\right]$ and $\left[\mu_{p}, \mu_{p}\right]_{p}=e_{3}(3)\left[\mu_{p}, \mu_{p}\right]$.

After applying the differentials $d$ on the double complex 1 , we obtain for $j, k \geq 1$ the first spectral sequence

$$
E_{1}^{j, k}=H_{(j)}^{j+k-1}(A) \Rightarrow H^{j+k-1}\left(C_{p}^{\bullet}(A)[1]\right),
$$

where $d_{1}=-\left[\mu_{p}, \cdot\right]: E_{1}^{j, k} \rightarrow E_{1}^{j+1, k}$.

To show that the functor $\operatorname{PDef}_{A}$ is controlled by the dgla $C_{p}^{\bullet}(A)[1]$ we first need few Lemmata. For a $k$-algebra $A$ we define the $k$-algebra $A_{0}$ that is as a $k$-vector space isomorphic to $A$ and it has zero multiplication.

Lemma 3.1. Poisson algebra structures on $A_{0}$ are in bijection with Maurer-Cartan elements of $C_{p}^{\bullet}\left(A_{0}\right)[1]$, i.e with elements $\left(\mu, \mu_{p}\right) \in C_{(1)}^{2}\left(A_{0}\right) \oplus C_{(2)}^{2}\left(A_{0}\right)$ satisfying $\frac{1}{2}[\mu, \mu]=\left[\mu, \mu_{p}\right]=\frac{1}{2}\left[\mu_{p}, \mu_{p}\right]_{p}=0$.

Proof. Let $\left(\mu, \mu_{p}\right)$ be a Maurer-Cartan element of $C_{p}^{\bullet}\left(A_{0}\right)[1]$. We define the multiplication on $A_{0}$ by $a \cdot b:=\mu(a, b):=\mu(a \otimes b)$ and the Poisson structure by $\{a, b\}:=\mu_{p}(a, b):=\mu_{p}(a \otimes b)$. The product $\cdot$ is commutative and associative if and only if $\mu \in C_{(1)}^{2}\left(A_{0}\right)$ and $\frac{1}{2}[\mu, \mu]=0$. Now we show that $\mu_{p}$ defines a Poisson structure. Since $\mu_{p} \in C_{(2)}^{2}\left(A_{0}\right)$, everything except the Jacobi identity is clear. The Jacobi identity we get from $\frac{1}{2}\left[\mu_{p}, \mu_{p}\right]_{p}=0$ as in Lemma 2.1 (note that we have $\left.\left[\mu_{p}, \mu_{p}\right]_{p}=e_{3}\left[\mu_{p}, \mu_{p}\right]\right)$. We now show the following claim:

$$
\left.\{a, b \cdot c\}=\{a, b\} c+\{a, c\} b \text { (i.e. } \mu_{p}(a, \mu(b, c))=\mu\left(\mu_{p}(a, b), c\right)+\mu\left(\mu_{p}(a, c), b\right)\right)
$$

holds if and only if $\left[\mu, \mu_{p}\right]=0$. Assume that

$$
F(a, b, c):=\mu_{p}(a, \mu(b, c))-\mu\left(\mu_{p}(a, b), c\right)-\mu\left(\mu_{p}(a, c), b\right)=0
$$

holds. We have

$$
\begin{aligned}
& F(a, b, c)+F(c, a, b)= \\
& \left(\mu_{p}(a, \mu(b, c))-\mu\left(\mu_{p}(a, b), c\right)-\mu\left(\mu_{p}(a, c), b\right)\right)+\left(\mu_{p}(c, \mu(a, b))-\mu\left(\mu_{p}(c, a), b\right)-\mu\left(\mu_{p}(c, b), a\right)\right)= \\
& -\left[\mu_{p}, \mu\right] .
\end{aligned}
$$

and thus we see one direction. For the other direction we compute

$$
\begin{aligned}
& {\left[\mu_{p}, \mu\right](a, b, c)+\left[\mu_{p}, \mu\right](a, c, b)-\left[\mu_{p}, \mu\right](b, a, c)=} \\
& \left(\mu_{p}(a b, c)-\mu_{p}(a, b c)+\mu_{p}(a, b) c-\mu_{p}(b, c) a\right)+\left(\mu_{p}(a c, b)-\mu_{p}(a, c b)+\mu_{p}(a, c) b-\mu_{p}(c, b) a\right)- \\
& \left(\mu_{p}(b a, c)-\mu_{p}(b, a c)+\mu_{p}(b, a) c-\mu_{p}(a, c) b\right)= \\
& 2\left(-\mu_{p}(a, b c)+\mu_{p}(a, b) c+\mu_{p}(a, c) b\right)=-2 F(a, b, c) .
\end{aligned}
$$

To shorten the notation we wrote $a b=\mu(a, b)$ and similarly for other elements.

Thus the claim is proved. From this we easily conclude the proof.

Definition 6. The Poisson product on a vector space $V$ is a pair $(\cdot,\{\}$,$) , such$ that $(V, \cdot,\{\}$,$) is a Poisson algebra.$

Lemma 3.2. Let $A$ be a Poisson algebra and let $B$ be an Artin ring. MaurerCartan elements of $C_{p}^{\bullet}\left(A \otimes m_{B}\right)[1]$ are in bijection with Poisson products on the vector space $A \otimes_{k} B$, giving the known Poisson product on $A \cong A \otimes_{k} B / m_{B}$. 
Proof. Let a Maurer-Cartan element $\left(\mu, \mu_{p}\right)$ of $C_{p}^{\bullet}\left(A_{0}\right)[1]$ represents the Poisson bracket of $A$. The Poisson products on the vector space $A \otimes_{k} B$, giving the known product on $A \cong A \otimes_{k} B / m_{B}$ are obtained by $\left(\xi, \xi_{p}\right) \in C_{(1)}^{2}\left(A \otimes m_{B}\right) \oplus C_{(2)}^{2}\left(A \otimes m_{B}\right)$ satisfying

$$
\left[\left(\mu, \mu_{p}\right)+\left(\xi, \xi_{p}\right),\left(\mu, \mu_{p}\right)+\left(\xi, \xi_{p}\right)\right]_{p}=0 .
$$

Since $\left[\left(\mu, \mu_{p}\right),\left(\mu, \mu_{p}\right)\right]_{p}=0$ and the differential on $C_{p}^{\bullet}\left(A \otimes m_{B}\right)[1]$ is given by $\left[\left(\mu, \mu_{p}\right), \cdot\right]$, then we see that the equation (2) gives us $\mathrm{MC}$ elements $\left(\xi, \xi_{p}\right)$ of $C_{p}^{\bullet}\left(A \otimes m_{B}\right)[1]$.

Proposition 3.3. For a Poisson algebra $A$ the functor PDef $_{A}$ is controlled by the $\operatorname{dgla} C_{p}^{\bullet}(A)[1]$.

Proof. We write for short $\mathfrak{g}:=C_{p}^{\bullet}(A)[1]$. By Lemma 3.2 there exists a bijection between $\mathrm{MC}_{\mathfrak{g}}(B)$ and Poisson products on the vector space $A \otimes_{k} B$, giving the known Poisson product on $A \cong A \otimes_{k} B / m_{B}$.

To conclude the proof we show that two Poisson products $(\cdot,\{\}$,$) and \left(.^{\prime},\{,\}^{\prime}\right)$ on $A \otimes_{k} B$ are equivalent (in the sense of Definition 5) if and only if the corresponding elements $\left(\gamma, \gamma_{p}\right),\left(\gamma^{\prime}, \gamma_{p}^{\prime}\right) \in \mathrm{MC}_{\mathfrak{g}}(B)$ are gauge equivalent. The products are equivalent if and only if there exists $\alpha \in C^{1}(A) \otimes m_{B}$ such that

$$
\begin{gathered}
a \cdot^{\prime} b=\exp (\alpha)(\exp (-\alpha)(a) \cdot \exp (-\alpha)(b)), \\
\{a, b\}^{\prime}=\exp (\alpha)(\{\exp (-\alpha)(a), \exp (-\alpha)(b)\}) .
\end{gathered}
$$

As above let a Maurer-Cartan element $\left(\mu, \mu_{p}\right)$ of $C_{p}^{\bullet}\left(A_{0}\right)[1]$ represents the Poisson bracket of $A$.

From (3) we obtain

$$
\left(\mu+\gamma^{\prime}\right)(a, b)=\exp (\alpha)(\exp (-\alpha)(a) \cdot \exp (-\alpha)(b))=\exp ([\alpha, \cdot])(\mu+\gamma)(a, b),
$$

where the later equality we get after some elementary computation. In the same way from (4) we obtain

Elements $\left(\gamma, \gamma_{p}\right) \in \mathrm{MC}_{\mathfrak{g}}(B)$ and $\left(\gamma^{\prime}, \gamma_{p}^{\prime}\right) \in \mathrm{MC}_{\mathfrak{g}}(B)$ are gauge equivalent if

$$
\left(\gamma^{\prime}, \gamma_{p}^{\prime}\right)=\left(\gamma, \gamma_{p}\right)+\sum_{n=0}^{\infty} \frac{[\alpha, \cdot]_{p}^{n}}{(n+1) !}\left(\left[\alpha,\left(\gamma, \gamma_{p}\right)\right]_{p}-\tilde{d}(\alpha)\right)
$$

holds.

Since $\tilde{d}(\alpha)=\left[\left(\mu, \mu_{p}\right), \alpha\right]_{p}=-\left[\alpha,\left(\mu, \mu_{p}\right)\right]_{p}$ and $[\alpha, \cdot]=[\alpha, \cdot]_{p}$, we see that (7) holds if and only if the equations (5) and (6) hold.

\section{Computation of the Hochschild and Poisson COHOMOlogy groups FOR GORENSTEIN TORIC VARIETIES}

4.1. Affine Gorenstein toric varieties. Let $M, N$ be mutually dual, finitely generated, free Abelian groups. We denote by $M_{\mathbb{R}}, N_{\mathbb{R}}$ the associated real vector spaces obtained via base change with $\mathbb{R}$. Let $\sigma=\left\langle a_{1}, \ldots, a_{N}\right\rangle \subset N_{\mathbb{R}}$ be a rational, polyhedral cone with apex in 0 and let $a_{1}, \ldots, a_{N} \in N$ denote its primitive fundamental generators (i.e. none of the $a_{i}$ is a proper multiple of an element of $N$ ). We define the dual cone $\sigma^{\vee}:=\left\{r \in M_{\mathbb{R}} \mid\langle\sigma, r\rangle \geq 0\right\} \subset M_{\mathbb{R}}$ and denote by $\Lambda:=\sigma^{\vee} \cap M$ the 
resulting semi-group of lattice points. Its spectrum $\operatorname{Spec}(k[\Lambda])$ is called an affine toric variety.

Affine toric Gorenstein varieties are obtained by putting a lattice polytope $P \subset$ $\mathbb{A} \cong \mathbb{R}^{n-1}$ into the affine hyperplane $\mathbb{A} \times\{1\} \subset \mathbb{A} \times \mathbb{R}=: N_{\mathbb{R}}$ and defining $\sigma:=$ Cone $(P)$, the cone over $P$. Then the canonical degree $R^{*} \in M$ equals $(\underline{0}, 1)$.

It is a trivial check that Hochschild differentials respect the grading given by the degrees $R \in M$. Thus we get the Hochschild subcomplex $C_{(i)}^{\bullet, R}$ and we denote the corresponding cohomology groups by $H_{(i)}^{n, R}(A) \cong T_{(i)}^{n-i, R}(A)$, where the later is the degree $R$ part of the (higher) André-Quillen cohomology group $T_{(i)}^{n-i, R}(A)$ (see [3, Section 4]). We will not use general André-Quillen cohomology theory, we will only use the well-known isomorphism $T_{(i)}^{n-i}(A) \cong H_{(i)}^{n}(A)$ for $n \geq i$ (see e.g. [8]).

4.2. Poisson cohomology groups of Poisson Gorenstein toric surfaces. Let $X_{\sigma_{n}}=\operatorname{Spec}\left(A_{n}\right)$ be the Gorenstein toric surface given by $g(x, y, z)=x y-z^{n+1}$. $\Lambda_{n}:=\sigma_{n}^{\vee} \cap M$ is generated by $S_{1}:=(0,1), S_{2}:=(1,1)$ and $S_{3}:=(n+1, n)$, with the relation $S_{1}+S_{3}=(n+1) S_{2}$.

In order to compute the Poisson cohomology groups we need to analyse the spectral sequence (11). First we need to understand all the parts of the Hochschild cohomology.

Proposition 4.1. It holds that

$$
\operatorname{dim}_{k} T_{(1)}^{1,-R}\left(A_{n}\right)=\operatorname{dim}_{k} T_{(2)}^{1,-R}\left(A_{n}\right)= \begin{cases}1 & \text { if } R=k S_{2} \text { for } 2 \leq k \leq n+1 \\ 0 & \text { otherwise. }\end{cases}
$$

Moreover, $T_{(1)}^{2}\left(A_{n}\right) \cong H_{(1)}^{3}\left(A_{n}\right)=0$. For $i \geq 3$ we have $T_{(i)}^{k}\left(A_{n}\right)=0$ if $k \neq i-1, i$ and

$$
T_{(i)}^{i-1}\left(A_{n}\right) \cong T_{(i)}^{i}\left(A_{n}\right) \cong A_{n} /\left(\frac{\partial g}{\partial x_{1}}, \frac{\partial g}{\partial x_{2}}, \frac{\partial g}{\partial x_{3}}\right) .
$$

The later has $k$-dimension equal to $n$.

Proof. [3, Proposition 3.3, Example 3].

Corollary 4.2. Since $T_{(i)}^{i-1}\left(A_{n}\right) \cong H_{(i)}^{2 i-1}\left(A_{n}\right)$ and $T_{(i)}^{i}\left(A_{n}\right) \cong H_{(i)}^{2 i}\left(A_{n}\right)$ we see that $E_{2}^{j, k}=E_{\infty}^{j, k}$ holds for every $j, k \geq 1$.

Elements from $H_{(2)}^{2}\left(A_{n}\right)$ define Poisson structures on $\operatorname{Spec}\left(A_{n}\right)$ by Lemma 2.1 , since $H_{(3)}^{3}(A)=0$. Let $\mu_{p} \in H_{(2)}^{2}\left(A_{n}\right)$ denote a Poisson structure on $\operatorname{Spec}\left(A_{n}\right)$. Let $\mathfrak{g}_{n}:=C_{p}\left(A_{n}\right)[1]$. From above we have the following description of the spectral sequence (11):

$$
\begin{aligned}
& E_{1}^{3, \bullet}: 0 \stackrel{d_{1}}{\longrightarrow} H_{(2)}^{4}\left(A_{n}\right) \stackrel{d_{1}}{\longrightarrow} H_{3}^{5}\left(A_{n}\right) \stackrel{d_{1}}{\longrightarrow} 0 \stackrel{d_{1}}{\longrightarrow} \cdots \\
& E_{1}^{2, \bullet}: H_{(1)}^{2}\left(A_{n}\right) \stackrel{d_{1}}{\longrightarrow} H_{(2)}^{3}\left(A_{n}\right) \stackrel{d_{1}}{\longrightarrow} 0 \stackrel{d_{1}}{\longrightarrow} 0 \stackrel{d_{1}}{\longrightarrow} \cdots \\
& E_{1}^{1, \bullet}: H_{(1)}^{1}\left(A_{n}\right) \stackrel{d_{1}}{\longrightarrow} H_{(2)}^{2}\left(A_{n}\right) \stackrel{d_{1}}{\longrightarrow} 0 \stackrel{d_{1}}{\longrightarrow} 0 \stackrel{d_{1}}{\longrightarrow} \cdots,
\end{aligned}
$$

$E_{1}^{j, \bullet}$ for $j>3$ have only two non-vanishing terms $E_{1}^{j, j-1}=H_{(j)}^{2 j-2}\left(A_{n}\right)$ and $E_{1}^{j, j}=H_{(j)}^{2 j-1}\left(A_{n}\right)$. 


\section{Corollary 4.3.}

$$
\begin{gathered}
H^{0}\left(\mathfrak{g}_{n}\right) \cong \operatorname{ker}\left(H_{(1)}^{1}\left(A_{n}\right) \stackrel{d_{1}}{\longrightarrow} H_{(2)}^{2}\left(A_{n}\right)\right), \\
H^{1}\left(\mathfrak{g}_{n}\right) \cong \operatorname{coker}\left(H_{(1)}^{1}\left(A_{n}\right) \stackrel{d_{1}}{\longrightarrow} H_{(2)}^{2}\left(A_{n}\right)\right) \oplus \operatorname{ker}\left(H_{(1)}^{2}\left(A_{n}\right) \stackrel{d_{1}}{\longrightarrow} H_{(2)}^{3}\left(A_{n}\right)\right),
\end{gathered}
$$

for $k \geq 2$ we have

$$
H^{k}\left(\mathfrak{g}_{n}\right) \cong \begin{cases}\operatorname{coker}\left(H_{\left(\frac{k}{2}\right)}^{k}\left(A_{n}\right) \stackrel{d_{1}}{\longrightarrow} H_{\left(\frac{k}{2}+1\right)}^{k+1}\left(A_{n}\right)\right) & \text { if } k \text { is even } \\ \operatorname{ker}\left(H_{\left(\frac{k+1}{2}\right)}^{k+1}\left(A_{n}\right) \stackrel{d_{1}}{\longrightarrow} H_{\left(\frac{k+1}{2}+1\right)}^{k+2}\left(A_{n}\right)\right) & \text { if } k \text { is odd. }\end{cases}
$$

Proposition 4.4. It holds that $H^{2}\left(\mathfrak{g}_{n}\right) \cong A_{n} /\left(\frac{\partial g}{\partial x_{1}}, \frac{\partial g}{\partial x_{2}}, \frac{\partial g}{\partial x_{3}}\right)$.

Proof. From Corollary 4.3 we know that $H^{2}\left(\mathfrak{g}_{n}\right) \cong \operatorname{coker}\left(H_{(1)}^{2}\left(A_{n}\right) \stackrel{d_{1}}{\longrightarrow} H_{(2)}^{3}\left(A_{n}\right)\right)$. In [4] we proved that the Gerstenhaber product $H_{(1)}^{2}\left(A_{n}\right) \times H_{(2)}^{2}\left(A_{n}\right) \rightarrow H_{(2)}^{3}\left(A_{n}\right)$ is the zero map. Since by definition $d_{1}=-\left[\mu_{p}, \cdot\right]$, we see that $d_{1}$ is the zero map. Thus $H^{2}\left(\mathfrak{g}_{n}\right) \cong H_{(2)}^{3}\left(A_{n}\right) \cong A_{n} /\left(\frac{\partial g}{\partial x_{1}}, \frac{\partial g}{\partial x_{2}}, \frac{\partial g}{\partial x_{3}}\right)$.

Example 1. For every hypersurface given by a polynomial $g(x, y, z)$ in $k^{3}$, we can define a Poisson structure $\pi_{g}$ on the quotient $k[x, y, z] / g$, namely:

$$
\pi_{g}:=\partial_{x}(g) \partial_{y} \wedge \partial_{z}+\partial_{y}(g) \partial_{z} \wedge \partial_{x}+\partial_{z}(g) \partial_{x} \wedge \partial_{y},
$$

i.e., we contract the differential 1-form $d g$ to $\partial_{x} \wedge \partial_{y} \wedge \partial_{z}$. In the case of Gorenstein toric surfaces $X_{\sigma_{n}}=\operatorname{Spec}\left(A_{n}\right)$ we have that

$$
\pi_{g}=f_{0}\left(\lambda_{1}, \lambda_{2}\right) x^{-S_{2}+\lambda_{1}+\lambda_{2}}
$$

where $f_{0}$ is skew-symmetric and bi-additive with $f_{0}\left(S_{1}, S_{3}\right)=-(n+1)$ (see [3, Example 4]). Thus we see that $\pi_{g} \in H_{(2)}^{2,-S_{2}}\left(A_{n}\right)$. In this case we see that $H^{1}\left(\mathfrak{g}_{n}\right) \cong$ $H^{2}\left(\mathfrak{g}_{n}\right) \cong A_{n} /\left(\frac{\partial g}{\partial x_{1}}, \frac{\partial g}{\partial x_{2}}, \frac{\partial g}{\partial x_{3}}\right)$ by the proof of Proposition 4.4 and Corollary 4.3 since $H_{(1)}^{1}\left(A_{n}\right) \stackrel{d_{1}}{\longrightarrow} H_{(2)}^{2}\left(A_{n}\right)$ is surjective. This is special case of [7, Lemma 3.1].

4.3. The Hochschild cohomology of three dimensional affine Gorenstein toric varieties. For an affine Gorenstein toric variety $X_{\sigma}=\operatorname{Spec}(A)$ we will explicitly compute $T_{(i)}^{1}(A)$ for all $i \geq 1$. They appear in $E_{1}^{\bullet, 2}$ (see (1) and note that $\left.H_{(j)}^{j+1}(A) \cong T_{(j)}^{1}(A)\right)$ and they are also important ingredients for understanding $\mathrm{HH}^{2}(A)$ and $\mathrm{HH}^{3}(A)$ (see Corollary 4.12). This subsection reproves and generalizes [1, Theorem 4.1].

In [3] we obtained a convex geometric description of $T_{(i)}^{1}(A)$ for $i \geq 1$, which we recall now. Let the cone $\sigma=\left\langle a_{1}, \ldots, a_{N}\right\rangle$ represent an $n$-dimensional toric variety $X_{\sigma}=\operatorname{Spec}(A), n \geq 3$. For $R \in M$ we define the affine space

$$
\mathbb{A}(R):=\left\{a \in N_{\mathbb{R}} \mid\langle a, R\rangle=1\right\} \subset N_{\mathbb{R}}
$$

and consider the polyhedron $Q(R):=\sigma \cap \mathbb{A}(R) \subset \mathbb{A}(R)$. Vertices of $Q(R)$ are $\bar{a}_{j}:=a_{j} /\left\langle a_{j}, R\right\rangle$, for all $j$ satisfying $\left\langle a_{j}, R\right\rangle \geq 1$. We denote $T_{(i)}^{1}(-R):=T_{(i)}^{1,-R}(A)$.

Let $d_{j k}:=\overline{a_{j} \bar{a}_{k}}$ denote the compact edges of $Q(R)$ (for $\left\langle a_{j}, a_{k}\right\rangle \leq \sigma,\left\langle a_{j}, R\right\rangle \geq 1$, $\left.\left\langle a_{k}, R\right\rangle \geq 1\right)$. We denote the lattice $N \cap \operatorname{Span}_{k}\left\langle a_{j}, a_{k}\right\rangle$ by $\bar{N}_{j k}$ and its dual with $\bar{M}_{j k}$. Let $\bar{R}_{j k}$ denote the projection of $R$ to $\bar{M}_{j k}$. By $T_{\left\langle a_{j}, a_{k}\right\rangle}^{1}\left(-\bar{R}_{j k}\right)$ we denote the degree $-\bar{R}_{j k}$ part of the toric surface given by a cone $\left\langle a_{j}, a_{k}\right\rangle$. We define $\operatorname{Span}_{k} K_{j k}^{R}$ to be

$$
\operatorname{Span}_{k} K_{j k}^{R}:=\operatorname{Span}_{k}\left(K_{a_{j}}^{R} \cap K_{a_{k}}^{R}\right),
$$


with $K_{a_{j}}^{R}=\left\{r \in \Lambda \mid\left\langle a_{j}, r\right\rangle<\left\langle a_{j}, R\right\rangle\right\}$. Let

$$
W_{j}(R):=\left\{\begin{array}{cc}
2 & \text { if }\left\langle a_{j}, R\right\rangle>1 \\
1 & \text { if }\left\langle a_{j}, R\right\rangle=1 \\
0 & \text { if }\left\langle a_{j}, R\right\rangle \leq 0
\end{array}\right.
$$

Proposition 4.5. If the compact part of $Q(R)$ lies in a two-dimensional affine space we have

$$
\operatorname{dim}_{k} T_{(i)}^{1}(-R)=\max \left\{0, \sum_{j=1}^{N} V_{j}^{i}(R)-\sum_{d_{j k} \in Q(R)} Q_{j k}^{i}(R)-\left(\begin{array}{c}
n \\
i
\end{array}\right)+s_{Q(R)}^{i}\right\},
$$

where

$$
\begin{aligned}
& V_{j}^{i}(R):= \begin{cases}\left(\begin{array}{c}
n \\
i
\end{array}\right) & \text { if }\left\langle a_{j}, R\right\rangle>1 \\
\left(\begin{array}{c}
n-1 \\
i
\end{array}\right) & \text { if }\left\langle a_{j}, R\right\rangle=1 \\
0 & \text { if }\left\langle a_{j}, R\right\rangle \leq 0,\end{cases}
\end{aligned}
$$

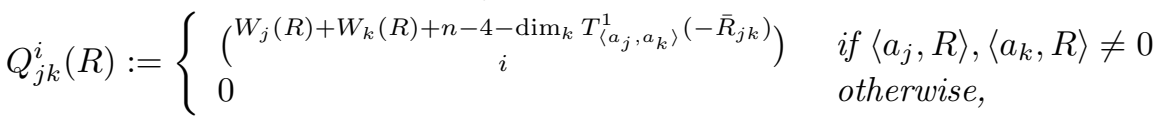

$$
\begin{aligned}
& s_{Q(R)}^{i}:= \begin{cases}\operatorname{dim}_{k} \wedge^{i}\left(\bigcap_{d_{j k} \in Q(R)} \operatorname{Span}_{k} K_{j k}^{R}\right) & \text { if } Q(R) \text { is compact } \\
0 & \text { otherwise. }\end{cases}
\end{aligned}
$$

Proof. See [3, Proposition 4.14].

From now on we assume that $X_{\sigma}$ is a three-dimensional affine toric Gorenstein variety given by a cone $\sigma=\left\langle a_{1}, \ldots, a_{N}\right\rangle$, where $a_{1}, \ldots, a_{N}$ are arranged in a cycle. Let $d_{j}:=a_{j+1}-a_{j}\left(a_{N+1}:=a_{1}\right)$ and let $\ell(j):=\ell\left(d_{j}\right)$ denote its lattice lenght. Let $s_{1}, \ldots, s_{N}$ be the fundamental generators of the dual cone $\sigma^{\vee}$, labelled so that $\sigma \cap\left(s_{j}\right)^{\perp}$ equals the face spanned by $a_{j}, a_{j+1} \in \sigma$. Using the previous notation we see that the polytope $P=Q\left(R^{*}\right)$ is a polygon with (oriented) edges equal to $d_{j}$ for $j=1, \ldots, N$. It holds that $\left\langle a_{j}, R^{*}\right\rangle=1$ for all $j=1, \ldots, N$.

Example 2. A typical example of a non-isolated, three dimensional toric Gorenstein singularity is the affine cone $X_{\sigma}$ over the weighted projective space $\mathbb{P}(1,2,3)$. The cone $\sigma$ is given by $\sigma=\left\langle a_{1}, a_{2}, a_{3}\right\rangle$, where

$$
a_{1}=(-1,-1,1), \quad a_{2}=(2,-1,1), \quad a_{3}=(-1,1,1) .
$$

We obtain $\sigma^{\vee}=\left\langle s_{1}, s_{2}, s_{3}\right\rangle$ with

$$
s_{1}=(0,1,1), \quad s_{2}=(-2,-3,1), \quad s_{3}=(1,0,1) .
$$

We need to better understand $s_{Q(R)}^{i}$ and $\operatorname{dim}_{k} T_{\left\langle a_{j}, a_{j+1}\right\rangle}^{1}\left(-\bar{R}_{j k}\right)$ that appears in $Q_{j, j+1}^{i}(R)$.

Lemma 4.6. Let a cone $\sigma=\left\langle a_{j}, a_{j+1}\right\rangle \subset \bar{N}_{j, j+1}$ define a toric surface given by the edge $d_{j}$. We have

$$
\operatorname{dim}_{k} \operatorname{Span}_{k} K_{j, j+1}^{R}=\max \left\{0, W_{j}(R)+W_{j+1}(R)-2-\operatorname{dim}_{k} T_{\left\langle a_{j}, a_{j}\right\rangle}^{1}\left(-\bar{R}_{j, j+1}\right)\right\} .
$$

Proof. See [3, Lemma 4.3].

\section{Lemma 4.7.}

$$
\operatorname{dim}_{k} T_{\left\langle a_{j}, a_{j+1}\right\rangle}^{1}\left(-\bar{R}_{j, j+1}\right)=\left\{\begin{array}{cc}
1 & \text { if } 2 \leq\left\langle a_{j}, R\right\rangle=\left\langle a_{j+1}, R\right\rangle \leq \ell(j) \\
0 & \text { otherwise. }
\end{array}\right.
$$


Proof. The toric surface $\left\langle a_{j}, a_{j+1}\right\rangle$ is isomorphic to the Gorenstein toric surface $\operatorname{Spec}\left(A_{\ell(j)-1}\right)$ and then the proof follows from Proposition 4.1 (more precisely from equation (8) $)$.

Lemma 4.8. If there exists $a_{j}$ such that $\left\langle R, a_{j}\right\rangle \leq 0$, then $s_{Q(R)}^{i}=0$, otherwise $s_{Q(R)}^{i} \leq\left(\begin{array}{l}3 \\ i\end{array}\right)$.

Proof. Follows trivially from definitions.

The next lemma establishes a useful criterion when $T_{(i)}^{1}(-R)$ is zero in the Gorenstein three-dimensional case.

Lemma 4.9. Assume that $\left\langle a_{j}, R\right\rangle \neq\left\langle a_{j+1}, R\right\rangle$ for all $j=1, \ldots, N\left(a_{N+1}:=a_{1}\right)$. Then $T_{(i)}^{1}(-R)=0$.

Proof. We will use Proposition 4.5. By the assumption and Lemma 4.7 we know that $\operatorname{dim}_{k} T_{\left\langle a_{j}, a_{j+1}\right\rangle}^{1}\left(-\bar{R}_{j, j+1}\right)=0$ for all $j$. Since $\left\langle a_{j}, R\right\rangle=1$ for at most two $j \in\{1, \ldots, N\}$ we can using Lemma 4.8 easily see that

$$
\sum_{j=1}^{N} V_{j}^{i}(R)-\sum_{j=1}^{N}\left(\begin{array}{c}
W_{j}(R)+W_{j+1}(R)-1 \\
i
\end{array}\right)-\left(\begin{array}{l}
3 \\
i
\end{array}\right)+s_{Q(R)}^{i} \leq 0,
$$

which implies that $T_{(i)}^{1}(-R)=0$ for all $i$.

Lemma 4.10. Let $R=q R^{*}$ for $q \geq 2$. It holds that $\operatorname{dim}_{k} \cap_{j} \operatorname{Span}_{k} E_{j, j+1}^{R}=3$ if $\ell(j)<q$ for all $j$. Moreover, $\operatorname{dim}_{k} \cap_{j} \operatorname{Span}_{k} E_{j, j+1}^{R}=2$ if $\ell(j)<q$ for all $j$ except two (denoted by $j_{1}$ and $j_{2}$ ), for which it holds that $d_{j_{1}}$ and $d_{j_{2}}$ are parallel (the case $j_{1}=j_{2}$ is included). Otherwise it holds that $\operatorname{dim}_{k} \cap_{j} \operatorname{Span}_{k} K_{j, j+1}^{R}=1$.

Proof. By definition we easily see that

$$
\operatorname{Span}_{k} K_{j, j+1}^{R}=\left\{\begin{array}{cc}
M \otimes_{\mathbb{Z}} k & \text { if } \ell(j)<q \\
\operatorname{Span}_{k}\left\{a_{j}^{\perp} \cap a_{j+1}^{\perp}, R^{*}\right\} & \text { if } \ell(j) \geq q .
\end{array}\right.
$$

Since $\operatorname{Span}_{k}\left\{a_{j}^{\perp} \cap a_{j+1}^{\perp}, R^{*}\right\}=\left\{c \in M \otimes_{\mathbb{Z}} k \mid\left\langle c, a_{j}\right\rangle=\left\langle c, a_{j+1}\right\rangle\right\}$ we see that $\operatorname{Span}_{k}\left\{a_{j_{1}}^{\perp} \cap a_{j_{1}+1}^{\perp}, R^{*}\right\}=\operatorname{Span}_{k}\left\{a_{j_{2}}^{\perp} \cap a_{j_{2}+1}^{\perp}, R^{*}\right\}$ for $j_{1}, j_{2} \in\{1, . ., N\}$ if and only if $j_{1}=j_{2}$ or $d_{j_{1}}$ is parallel to $d_{j_{2}}$. Thus we can easily conclude the proof.

Let $\operatorname{int}\left(\sigma^{\vee}\right)$ denotes the interior of $\sigma^{\vee}$.

Theorem 4.11. Let $X_{\sigma}$ be a three-dimensional affine toric Gorenstein variety. The following holds:

(1) $T_{(1)}^{1}(-R)$ is non-trivial in the following cases:

(a) $R=R^{*}$ with $\operatorname{dim}_{k} T_{(1)}^{1}(-R)=N-3$,

(b) $R=q R^{*}\left(\right.$ for $q \geq 2$ ) with $\operatorname{dim}_{k} T_{(1)}^{1}(-R)=\max \{0, \#\{j \mid q \leq \ell(j)\}-2\}$,

(c) $R=q R^{*}-p s_{j}$ with $2 \leq q \leq \ell(j)$ and $p \in \mathbb{Z}$ sufficiently large such that $R \notin \operatorname{int}\left(\sigma^{\vee}\right)$. In this case $\operatorname{dim}_{k} T_{(1)}^{1}(-R)=1$.

Additional degrees exist only in the following two (overlapping) exceptional cases:

(d) $P$ contains a pair of parallel edges $d_{j}, d_{k}$, both longer than every other edge. Then $\operatorname{dim}_{k} T_{(1)}^{1}\left(-q R^{*}\right)=1$ for $q$ in the range

$$
\max \{\ell(l) \mid l \neq j, k\}<q \leq \min \{\ell(j), \ell(k)\}\},
$$


(e) $P$ contains a pair of parallel edges $d_{j}, d_{k}$ (with distance $d=\left\langle a_{j}, s_{k}\right\rangle=$ $\left.\left\langle a_{k}, s_{j}\right\rangle\right)$. In this case $\operatorname{dim}_{k} T_{(1)}^{1}(-R)=1$ for $R=q R^{*}+p s_{j}$ with $1 \leq q \leq \ell(j)$ and $1 \leq p \leq(\ell(k)-q) / d$.

(2) $T_{(2)}^{1}(-R)$ is non-trivial in the following cases:

(a) $R=R^{*}$ with $\operatorname{dim}_{k} T_{(2)}^{1}(-R)=N-3$,

(b) $R=q R^{*}($ for $q \geq 2)$ with $\operatorname{dim}_{k} T_{(2)}^{1}(-R)=\max \{0,2 \cdot \#\{j \mid q \leq$ $\ell(j)\}-3\}$,

(c) $R=q R^{*}-p s_{j}$ with $2 \leq q \leq \ell(j)$ and $p \in \mathbb{Z}$ sufficiently large such that $R \notin \operatorname{int}\left(\sigma^{\vee}\right)$. In this case $\operatorname{dim}_{k} T_{(2)}^{1}(-R)=2$.

Additional degrees exist only in the following two (overlapping) exceptional cases:

(d) $P$ contains a pair of parallel edges $d_{j}, d_{k}$, both longer than every other edge. Then $\operatorname{dim}_{k} T_{(2)}^{1}\left(-q R^{*}\right)=2$ for $q$ in the range

$$
\max \{\ell(l) \mid l \neq j, k\}<q \leq \min \{\ell(j), \ell(k)\}\},
$$

(e) $P$ contains a pair of parallel edges $d_{j}, d_{k}$ (with distance $d=\left\langle a_{j}, s_{k}\right\rangle=$ $\left.\left\langle a_{k}, s_{j}\right\rangle\right)$. In this case $\operatorname{dim}_{k} T_{(2)}^{1}(-R)=2$ for $R=q R^{*}+p s_{j}$ with $1 \leq q \leq \ell(j)$ and $1 \leq p \leq(\ell(k)-q) / d$.

(3) $T_{(3)}^{1}(-R)$ is non-trivial in the following cases:

(b) $R=q R^{*}$ (for $q \geq 2$ ) with $\operatorname{dim}_{k} T_{(3)}^{1}(-R)=\max \{0, \#\{j \mid q \leq \ell(j)\}-1\}$,

(c) $R=q R^{*}-p s_{j}$ with $2 \leq q \leq \ell(j)$ and $p \in \mathbb{Z}$ sufficiently large such that $R \notin \operatorname{int}\left(\sigma^{\vee}\right)$. In this case $\operatorname{dim}_{k} T_{(3)}^{1}(-R)=1$.

Additional degrees exist only in the following two (overlapping) exceptional cases:

(d) $P$ contains a pair of parallel edges $d_{j}, d_{k}$, both longer than every other edge. Then $\operatorname{dim}_{k} T_{(3)}^{1}\left(-q R^{*}\right)=1$ for $q$ in the range

$$
\max \{\ell(l) \mid l \neq j, k\}<q \leq \min \{\ell(j), \ell(k)\}\},
$$

(e) $P$ contains a pair of parallel edges $d_{j}, d_{k}$ (with distance $d=\left\langle a_{j}, s_{k}\right\rangle=$ $\left.\left\langle a_{k}, s_{j}\right\rangle\right)$. In this case $\operatorname{dim}_{k} T_{(3)}^{1}(-R)=1$ for $R=q R^{*}+p s_{j}$ with $1 \leq q \leq \ell(j)$ and $1 \leq p \leq(\ell(k)-q) / d$.

(4) We have $T_{(i)}^{1}(-R)=0$ for $i \geq 4$.

Proof. We distinguish the following cases.

- Let $R=R^{*}$. In this case we have $\left\langle a_{j}, R\right\rangle=1$ and $\operatorname{Span}_{k} K_{a_{j}}^{R}=\left(a_{j}\right)^{\perp}$ for all $j$. Thus $s_{Q(R)}^{i}=0$ for all $i$ and by Lemma 4.7 we have $T_{\left\langle a_{j}, a_{j+1}\right\rangle}^{1}\left(-\bar{R}_{j, j+1}\right)=0$ for all $j$. Moreover,

$$
\sum_{j=1}^{N} V_{j}^{i}(R)-\sum_{d_{j k} \in Q(R)} Q_{j k}^{i}(R)=N\left(\begin{array}{l}
2 \\
i
\end{array}\right)-N\left(\begin{array}{l}
1 \\
i
\end{array}\right) .
$$

From Proposition 4.5 it follows that $\operatorname{dim}_{k} T_{(1)}^{1}\left(-R^{*}\right)=\operatorname{dim}_{k} T_{(2)}^{1}\left(-R^{*}\right)=$ $N-3$ and $T_{(i)}^{1}\left(-R^{*}\right)=0$ for $i>2$. Thus we proved (a) cases (note that $T_{(3)}^{1}\left(-R^{*}\right)=0$ and thus (a) case does not appear in the case (3)).

- Let $R=q R^{*}$, where $q \geq 2$.

In this case we have $\left\langle a_{j}, R\right\rangle \geq 2$. Thus $\sum_{j=1}^{N} V_{j}^{i}(R)=\left(\begin{array}{l}3 \\ i\end{array}\right) N$. Let us define $v:=\#\{j \mid q \leq \ell(j)\}$. 
For $i=1$ we have $\sum_{j=1}^{N} Q_{j, j+1}^{1}(R)=3 N-v$ (since for $q \leq \ell(j)$ we have $\operatorname{dim}_{k} T_{\left\langle a_{j}, a_{j+1}\right\rangle}^{1}\left(-\bar{R}_{j, j+1}\right)=1$ by Lemma 4.7). Thus $\operatorname{dim}_{k} T_{(1)}^{1}(-R)=$ $v-3+s_{Q(R)}^{1}$ holds by Proposition 4.5. If $s_{Q(R)}^{1}=1$ (see Lemma 4.10 when this holds) we obtain the case (1b). There is an exceptional case for which $\operatorname{dim}_{k} T_{(1)}^{1}(-R) \neq 0$ : this appears if $v=2$ and $s_{Q(R)}^{1}=2$. From Lemma 4.10 we see that this happens for $q$ in the range

$$
\max \{\ell(l) \mid l \neq j, k\}<q \leq \min \{\ell(j), \ell(k)\}\} .
$$

Thus we proved the case (1d).

In the case $i=2$ we have $\sum_{j=1}^{N} Q_{j, j+1}^{2}(R)=\left(\begin{array}{l}2 \\ 2\end{array}\right) v+\left(\begin{array}{l}3 \\ 2\end{array}\right)(N-v)=3 N-2 v$. Thus Proposition 4.5 gives us that $\operatorname{dim}_{k} T_{(2)}^{1}(-R)=2 v-3+s_{Q(R)}^{2}$. As in the case $i=1$ we obtain from Lemma 4.10 the cases (2b) and (2d).

For $i=3$ we have $\sum_{j=1}^{N} Q_{j, j+1}^{3}(R)=N-v$. By Proposition 4.5 we have $\operatorname{dim}_{k} T_{(3)}^{1}(-R)=v-1+s_{Q(R)}^{3}$. As in the case $i=1$ we obtain from Lemma 4.10 the cases $(3 \mathrm{~b})$ and $(3 \mathrm{~d})$.

- Let $R \notin \operatorname{int}\left(\sigma^{\vee}\right)$. By Lemma 4.9 we see that the only possible cases for having a non-zero $T_{(i)}^{1}(-R)$ occur when $\left\langle a_{j}, R\right\rangle=\left\langle a_{j+1}, R\right\rangle>0$ for some $j \in\{1, \ldots, N\}$ and $\left\langle a_{l}, R\right\rangle \leq 0$ for all other $l$. This happens for $R=q R^{*}-p s^{j}$ with $q \geq 1$ and $p \in \mathbb{Z}$ sufficiently large such that $R \notin \operatorname{int}\left(\sigma^{\vee}\right)$. In this case we have $\left\langle a_{j}, R\right\rangle=\left\langle a_{j+1}, R\right\rangle=q$ and $\left\langle a_{l}, R\right\rangle \leq 0$ for other $l$. If $q=1$, then by Lemma 4.7 it holds that $T_{\left\langle a_{j}, a_{j+1}\right\rangle}^{1}\left(-\bar{R}_{j, j+1}\right)=0$ and thus by Proposition 4.5 we have

$$
\operatorname{dim}_{k} T_{(i)}^{1}(-R)=\max \left\{0,2\left(\begin{array}{l}
2 \\
i
\end{array}\right)-\left(\begin{array}{l}
1 \\
i
\end{array}\right)-\left(\begin{array}{l}
3 \\
i
\end{array}\right)\right\}=0
$$

for all $i$. If $q \geq 2$, then using Lemma 4.7 we see that

$$
\operatorname{dim}_{k} T_{(i)}^{1}(-R)=\left\{\begin{array}{cc}
2\left(\begin{array}{l}
3 \\
i
\end{array}\right)-\left(\begin{array}{l}
2 \\
i
\end{array}\right)-\left(\begin{array}{l}
3 \\
i
\end{array}\right) & \text { if } 2 \leq q \leq \ell(j) \\
2\left(\begin{array}{l}
3 \\
i
\end{array}\right)-\left(\begin{array}{l}
3 \\
i
\end{array}\right)-\left(\begin{array}{l}
3 \\
i
\end{array}\right)=0 & \text { if } q>\ell(j) .
\end{array}\right.
$$

In the cases $2 \leq q \leq \ell(j)$ we see that $\operatorname{dim}_{k} T_{(1)}^{1}(-R)=\operatorname{dim}_{k} T_{(3)}^{1}(-R)=1$, $\operatorname{dim}_{k} T_{(2)}^{1}(-R)=2$ and $\operatorname{dim}_{k} T_{(i)}^{1}(-R)=0$ for $i \geq 4$. This proves (c) cases.

- Let $R \in \operatorname{int}\left(\sigma^{\vee}\right)$ and $R \neq q R^{*}$ for some $q \geq 1$. By Lemma 4.9 it follows that $T_{(i)}^{1}(-R)=0$ for all $R$, except maybe for $R=q R^{*}+p s_{j}$ for some $j$ since in this case we have $\left\langle a_{j}, R\right\rangle=\left\langle a_{j+1}, R\right\rangle=q$.

Let us first assume that $q \geq 2$. In this case we have that

$$
\operatorname{dim}_{k} T_{(i)}^{1}(-R)=N\left(\begin{array}{l}
3 \\
i
\end{array}\right)-\sum_{l=1}^{N}\left(\begin{array}{c}
3-\operatorname{dim}_{k} T_{\left\langle a_{l}, a_{l+1}\right\rangle}^{1}\left(-\bar{R}_{l, l+1}\right) \\
i
\end{array}\right)-\left(\begin{array}{l}
3 \\
i
\end{array}\right)+s_{Q(R)}^{i} .
$$

We see that $T_{(i)}^{1}(-R)=0$ if $T_{\left\langle a_{l}, a_{l+1}\right\rangle}^{1}\left(-\bar{R}_{l, l+1}\right)=0$ for all $l \in\{1, \ldots, N\}$ since $s_{Q(R)}^{i} \leq\left(\begin{array}{l}3 \\ i\end{array}\right)$. If only $T_{\left\langle a_{j}, a_{j+1}\right\rangle}^{1}\left(-\bar{R}_{j, j+1}\right) \neq 0$ we still obtain $T_{(i)}^{1}(-R)=$ 0 since in this case $s_{Q(R)}^{i} \leq\left(\begin{array}{l}2 \\ i\end{array}\right)$ (by Lemma 4.6). Thus we see that the only case to obtain nontrivial $T_{(i)}^{1}(-R)$ is when there exist parallel edges $d_{j}, d_{k}$ (with distance $d=\left\langle a_{j}, s_{k}\right\rangle=\left\langle a_{k}, s_{j}\right\rangle$ ). We have $\operatorname{dim}_{k} T_{(i)}^{1}(-R) \neq 0$ for $R=q R^{*}+p s_{j}$ with $2 \leq q \leq \ell(j)$ and $1 \leq p \leq(\ell(k)-q) / d$ since in this case $\operatorname{dim}_{k} T_{\left\langle a_{j}, a_{j+1}\right\rangle}^{1}\left(-\bar{R}_{j, j+1}\right)=\operatorname{dim}_{k} T_{\left\langle a_{k}, a_{k+1}\right\rangle}^{1}\left(-\bar{R}_{j, j+1}\right)=1$ by Lemma 
4.7. Thus we have

$$
\operatorname{dim}_{k} T_{(i)}^{1}(-R)=N\left(\begin{array}{l}
3 \\
i
\end{array}\right)-\left(2\left(\begin{array}{l}
2 \\
i
\end{array}\right)+(N-2)\left(\begin{array}{l}
3 \\
i
\end{array}\right)\right)-\left(\begin{array}{l}
3 \\
i
\end{array}\right)+\left(\begin{array}{l}
2 \\
i
\end{array}\right)=\left(\begin{array}{l}
3 \\
i
\end{array}\right)-\left(\begin{array}{l}
2 \\
i
\end{array}\right) .
$$

We see that in this case $\operatorname{dim}_{k} T_{(1)}^{1}(-R)=\operatorname{dim}_{k} T_{(3)}^{1}(-R)=1$ and $\operatorname{dim}_{k} T_{(2)}^{1}(-R)=$ 2. Similarly we can treat the case $q=1$ and thus finish the proof.

Remark 2. Note that in the case $i=1$ our formulas agree with the ones given in [1, Theorem 4.1], which were obtained by different methods.

Example 3. Let $X_{\sigma}$ be as in Example 2, From Theorem 4.11 we obtain that if $R \in\left\{2 R^{*}-\alpha s_{3}, 2 R^{*}-\beta s_{1}, 2 R^{*}-\gamma s_{1} \mid \alpha \geq 1, \beta \geq 1, \gamma \geq 2\right\}$, then $\operatorname{dim}_{k} T_{(1)}^{1}(-R)=$ $\operatorname{dim}_{k} T_{(3)}^{1}(-R)=1$ and $\operatorname{dim}_{k} T_{(2)}^{1}(-R)=2$. For other degrees $S \in M$ we have $T_{(i)}^{1}(-S)=0$ for all $i \geq 1$.

Corollary 4.12. Let $\operatorname{Spec}(A)$ be a three-dimensional affine toric Gorenstein variety. The Hodge decomposition gives us

$$
\begin{gathered}
\operatorname{HH}^{2}(A) \cong T_{(1)}^{1}(A) \oplus T_{(2)}^{0}(A), \\
\operatorname{HH}^{3}(A) \cong T_{(1)}^{2}(A) \oplus T_{(3)}^{0}(A) \oplus T_{(2)}^{1}(A) .
\end{gathered}
$$

Descriptions of $T_{(2)}^{0}(A)$ and $T_{(3)}^{0}(A)$ were given in [3]. The module $T_{(1)}^{2}(A)$ was analysed in [2, Corollary 5.4]. Theorem 4.11 gives us an explicit description of $T_{(1)}^{1}(A)$ and $T_{(2)}^{1}(A)$ and thus we complete understanding of the second (which describes the first order associative non-commutative deformations) and third Hochschild cohomology group (which contains the obstructions for extending associative noncommutative deformations to larger base spaces).

\section{ACKNOWLEDGEMENTS}

I would like to thank to my $\mathrm{PhD}$ advisor Klaus Altmann for his constant support and many useful conversations.

\section{REFERENCES}

[1] K. Altmann: One parameter families containing three-dimensional toric Gorenstein singularities, Explicit birational geometry of 3-folds, London Math. Soc. Lecture Note Ser., vol. 281, Cambridge Univ. Press, Cambridge (2000), 21-50.

[2] K. Altmann, A. B. Sletsjøe: André-Quillen cohomology of monoid algebras, J. Alg. 210 (1998), 1899-1911.

[3] M. Filip: Hochschild cohomology and deformation quantization of affine toric varieties, J. Alg. 508 (2018), 188-214.

[4] M. Filip: The Gerstenhaber product $\mathrm{HH}^{2}(A) \times \mathrm{HH}^{2}(A) \rightarrow \mathrm{HH}^{3}(A)$ of affine toric varieties, arXiv:1803.07486

[5] B. Fresse: Homologie de Quillen pour les algebres de Poisson, C.R. Acas. Sci. Paris 326 (1998), 1053-1058.

[6] M. Gerstenhaber, S.D. Schack: A Hodge-type decomposition for commutative algebras, J. Pure Appl. Alg. 48 (1987), 229-247.

[7] V. Ginzburg, D. Kaledin: Poisson deformations of symplectic quotient singularities, Adv. in Math. 186, (2004), pp. 1-57.

[8] J.-L. Loday: Cyclic homology, Grundlehren der mathematischen Wissenschaften 301, Springer-Verlag, (1992).

[9] Y. Namikawa: Flops and Poisson deformations of symplectic varieties, Publ. RIMS, 44 (2008), 259-314. 
[10] Y. Namikawa: Poisson deformations of affine symplectic varieties Duke Math. J. 156 (2011) 51-85.

[11] Y. Namikawa: Poisson deformations and Birational Geometry, J. Math. Sci. Univ. Tokyo 22 (2015), 339-359.

[12] V.P. Palamodov: Infinitesimal deformation quantization of complex analytic spaces, Lett. Math. Phys. 79 (2007), iss. 2, 131-142.

JGU Mainz, Institut fur Mathematik, Staudingerweg 9, 55099 Mainz, Germany

E-mail address: mfilip@uni-mainz.de 